\title{
Current understanding of the pathophysiology of thrombotic thrombocytopenic purpura
}

\author{
S L Allford, S J Machin
}

Thrombotic thrombocytopenic purpura (TTP) was first described by Moschowitz in 1924. ${ }^{1}$ Although it is now well recognised, being characterised by the classic pentad of fever, thrombocytopenia, microangiopathic haemolytic anaemia (MAHA), renal impairment, and fluctuating neurological signs, its precise pathophysiology remains elusive. Several other syndromes including haemolytic uraemic syndrome (HUS), eclampsia and HELLP (haemolysis, elevated liver enzymes, and low platelets) are also characterised by MAHA and this has given rise to the concept that they might represent a spectrum of disease. However, recent independent work by Tsai and Furlan indicates that TTP and HUS appear to be distinct pathophysiological entities, which might explain in part the wide variation in response to treatment that is seen in clinical practice.

\section{Clinical variants}

Several variants of TTP are recognised. Most cases are defined as single episode TTP: in these patients there is no identifiable precipitant and no subsequent recurrence. Because survival has improved with advances in treatment, it is now apparent that a few patients continue to relapse at infrequent intervals: in one study it was estimated that $11-36 \%$ fall into this category, known as intermittent TTP (fig 1), with relapses occurring up to eight years after the index episode. ${ }^{2}$ In some instances, the index or subsequent episode might be precipitated by an identifiable cause, resulting in secondary TTP. Several drugs have been implicated including mitomycin $\mathrm{C}$, ticlopidine, cyclosporin, oral contraceptives, and quinine. Perhaps those most closely linked are ticlopidine and cyclosporin. Ticlopidine characteristically results in TTP after two to four weeks of use, ${ }^{3}$ whereas cyclosporin is a recognised risk factor in post-allogeneic bone marrow transplant associated TTP, along with total body irradiation conditioning. Pregnancy and systemic lupus erythematosus (SLE) are other associations and might account, in part, for the female preponderance (male to female ratio, $1: 2$ ). Anecdotally, TTP also appears to be triggered by viral infections. However, because fever is one constituent of the defining pentad of TTP, such symptoms might actually represent a prodromal TTP illness. Nevertheless, certain infections are associated: HIV infection, especially when associated with a high viral load, is one example. Of interest, a TTP like illness in association with Bartonella bacilliformis like organisms adherent to $0.1-2 \%$ of circulating erythrocytes has been reported in five patients. ${ }^{4}$ It would appear, however, that

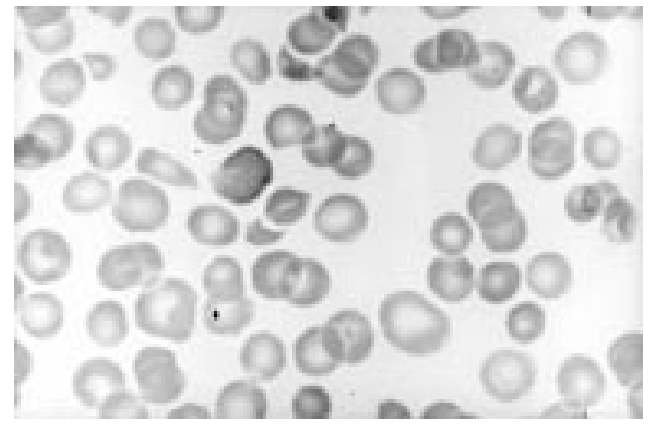

Figure 1 Peripheral blood film of patient with intermittent thrombotic thrombocytopenic purpura (TTP). Severe

thrombocytopenia and red cell fragmentation are prominent features. Circulating normoblasts and neutrophilia may also be seen.

infectious precipitants are relatively uncommon because TTP is not associated with seasonal variation and case clustering rarely occurs. Further research into this area is awaited.

Rarely, despite treatment, there is evidence of continued haemolysis, as shown by persistently raised lactate dehydrogenase $(\mathrm{LDH})$ in combination with a reticulocytosis and thrombocytopenia. This is classified as chronic unremitting TTP, which should be differentiated from chronic relapsing TTP. The latter is a congenital condition usually presenting in infancy or early childhood and is characterised by frequent episodes of haemolysis at predictable intervals (usually every 21-30 days). In contrast to single episode or intermittent TTP, these episodes normally respond to infusion of fresh frozen plasma and there is no requirement for concurrent plasmapheresis. ${ }^{5}$

\section{Histology}

Regardless of the exact nature of TTP, be it congenital or acquired, the histological findings appear to be identical. Microvascular thrombi are the predominant abnormality, and are found primarily in the renal and cerebral circulation, thus accounting for the clinical features of the disease (fig 2). However, other anatomical sites can be affected-for example, abdominal pain and serous retinal detachment are recognised complications of TTP and are thought to be secondary to mesenteric and retinal ischaemia, respectively.

The microvascular thrombi formed have been shown to be composed almost entirely of platelets. Because neither pronounced perivascular inflammation, obvious endothelial cell desquamation, nor subendothelial exposure have been demonstrated in TTP, it has been hypothesised that TTP might be a disease of primary platelet aggregation within the 


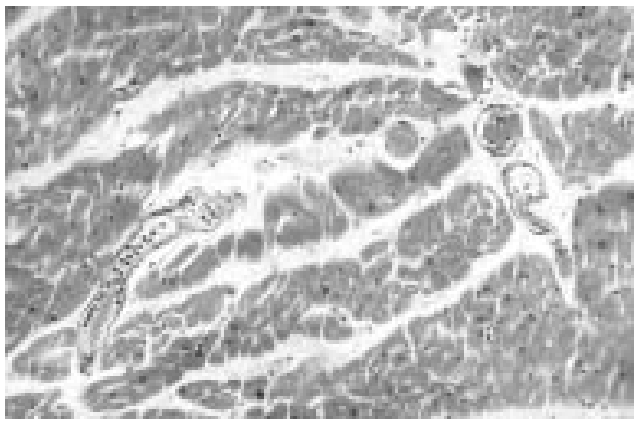

Figure 2 Typical histological findings of acute thrombotic thrombocytopenic purpura (TTP) (cardiac muscle). There is florid thrombosis within the microvasculature.

Immunohistochemistry shows these thrombi to be composed predominantly of von Willebrand factor (vWF) and platelets, with only small amounts of fibrin and fibrinogen.

microvasculature. ${ }^{6}$ In support of this, immunohistochemistry shows that thrombi formed in TTP contain abundant amounts of von Willebrand factor (vWF) and little fibrinogen or fibrin, in contrast to those found in disseminated intravascular coagulation (DIC), where the reverse is seen. ${ }^{7}$ This has led to the hypothesis that vWF multimers, perhaps in conjunction with high shear stress, might promote platelet aggregation during episodes of TTP.

\section{Platelet aggregation}

Platelets form aggregates through sequential stages of adhesion, activation, and aggregation. Although platelets circulate in close proximity to the endothelium, intravascular aggregation normally only occurs after endothelial damage. Subendothelial vWF is then exposed and binds to the platelet glycoprotein (Gp) Ib-IX-V receptor. This interaction has an extremely fast association rate and therefore proceeds despite the high shear pressures that exist within the microvasculature. Platelet activation results and, as a consequence, the platelet GpIIbIIIa receptor undergoes a conformational change, which allows binding not only to surface bound fibrinogen, but also to free fibrinogen. Crosslinkage of platelets follows, secondary to the dimeric nature of fibrinogen, and aggregation results. However, if platelets are exposed to high shear pressures, direct platelet aggregation may occur in the fluid phase. This phenomenon has an absolute requirement for $\mathrm{vWF}$, GpIb, GpIIbIIIa, calcium, and ADP..$^{8-10}$

Evidence for the importance of direct platelet aggregation in the pathogenesis of TTP has been provided experimentally: a cone and plate viscometer minimises platelet surface interactions and allows direct platelet aggregation under raised shear fluid stresses. Using this model, it has been demonstrated that platelet rich plasma from patients with congenital TTP results in excessive platelet aggregation, as compared with normal adult platelet rich plasma samples, when exposed to shear stresses of $90-180$ dyne $/ \mathrm{cm}^{2}$ (similar to those found in partially occluded microvasculature).$^{11}$ Mixing studies suggest that this is caused by a plasma component rather than being secondary to a platelet defect. More specifically, ultra large forms of vWF (ULvWF) are implicated because, not only are they present in TTP plasma (fig 3), but the addition of ULvWF to normal plasma accentuates platelet aggregation. Similarly, platelet retention, as measured by the modified Adeplat $S$ test (a glass bead retention method) at the relatively modest shear stress of $76 \mathrm{dyne} / \mathrm{cm}^{2}$, is increased in congenital TTP compared with normal controls. In this case, loss of ULvWF is associated with normalisation of increased platelet retention, again supporting the concept that vWF multimers might play an integral role in TTP. ${ }^{12}$

\section{Von Willebrand factor}

vWF circulates in the plasma as a series of multimers of $500-20000 \mathrm{kDa}$ and is composed of $270 \mathrm{kDa}$ monomers linked by disulphide bonds. Although it is synthesised in megakaryocytes and stored within platelet $\alpha$ granules, the predominant source of plasma vWF is the endothelium, where it is stored within the Wiebel-Palade bodies. ULvWF is found in endothelial cells and platelets and probably consists of an increased number of mature vWF subunits because pro-vWF monomers do not appear to be a constituent. ${ }^{13}$ ULvWF multimers are secreted in a retrograde direction from endothelial cells to the vascular subendothelium, where they are protected from proteolytic cleavage. If ULvWF do enter the circulation they have been shown to be superior to the largest plasma vWF forms at binding under the influence of fluid shear stress to platelet receptors GpIb-IX-V and GpIIbIIIa, and hence inducing platelet aggregation. ${ }^{8}$ Similarly, ULvWF forms have also been shown to be more effective than normal circulating vWF forms in supporting vWF attachment to a filter device. ${ }^{14}$ They are not, however, a normal constituent of circulating plasma: the smaller circulating multimeric forms are thought to result from proteolytic degradation of ULvWF.

\section{vWF proteolysis}

Evidence for vWF proteolysis has been provided by a number of groups: vWF fragments with mobility corresponding to $189 \mathrm{kDa}$, $176 \mathrm{kDa}$, and $140 \mathrm{kDa}$ are consistently detected in normal plasma in addition to the predominant $225 \mathrm{kDa}$ subunit. ${ }^{15}{ }^{16}$ It appears that circulating vWF multimers are originally composed of a varying number of intact $225 \mathrm{kDa}$ subunit dimers but proteolysis causes the appearance of two predominant fragments of $140 \mathrm{kDa}$ and $176 \mathrm{kDa}$. These originate as a consequence of cleavage of a single peptide bond between residues Tyr 842 and Met 843 of the mature subunit. ${ }^{17}$ However, the precise mechanism by which this is achieved is unknown. It has been shown in vitro that normal plasma and cryosupernatant contain a substance that reversibly reduces the size of ULvWF multimers released from cultured endothelial cells into smaller multimeric forms. This reversible reaction appears to have the characteristics of a limited disulphide bond reductase. ${ }^{18}$ Because ULvWF multimers are not a normal circulating constituent of plasma, 
it was hypothesised that this process might be rendered irreversible by the subsequent proteolysis of partially unfolded $\mathrm{vWF}$ multimers.

Although vWF is stable in plasma, multimeric degradation results in vitro either at low salt concentrations, a process that is promoted by the presence of urea, ${ }^{19}$ or after unfolding of vWF is induced by guanidine hydrochloride. ${ }^{20}$ Under both conditions the fragments generated, of molecular weight $140 \mathrm{kDa}$ and $170 \mathrm{kDa}$, are consistent with cleavage of the peptide bond between Tyr 842 and Met 843, suggesting that a physiological phenomenon is occurring. Using these models it has been shown that the vWF cleaving protease activity possesses several unusual properties. Unlike other known metalloenzymes, zinc ions are not required for full activity and barium rather than calcium ions activate the protease most efficiently. In addition, in contrast to acid lysosomal cathepsins the protease has an optimum $\mathrm{pH}$ of $8-10$. Partial purification has linked the proteolytic activity to a high molecular weight protein (approximately $300 \mathrm{kDa}$ ) and it has therefore been proposed that the enzyme might be complexed with, for instance, a plasma protein inhibitor. ${ }^{19}$

Although it has long been accepted that vWF function is affected by its multimeric size, the above studies also suggest that the conformation of the molecule is crucial in determining its proteolytic susceptibility. Exposure of tryptophan and/or tyrosine residues might be important because a shift in fluorescence to longer wavelengths is seen after incubation of vWF with guanidine $\mathrm{HCl}$. A similar shift in fluorescence intensity of up to $4 \mathrm{~nm}$ can also be

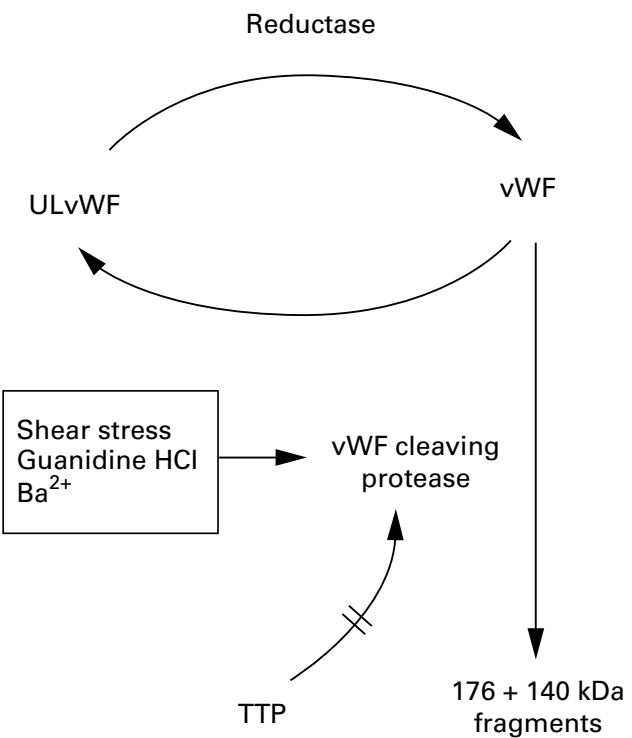

Figure 4 Proposed mechanism for proteolytic breakdown of von Willebrand factor ( $v W F$ ). Ultra large forms of $v W F$ (ULvWF) are reversibly converted in vitro to those forms normally found circulating in plasma by an activity in the cryosupernatant fraction of normal plasma with properties of a limited disulphide bond reductase. Under the conditions shown, a metalloproteinase further cleaves wWF forms into circulating $140 \mathrm{kDa}$ and $176 \mathrm{kDa}$ fragments, thus preventing reversible re-formation of ULvWF. This metalloproteinase activity has been reported to be absent in chronic relapsing thrombotic thrombocytopenic purpura (TTP), whereas it appears to be inhibited in single episode or intermittent adult TTP. observed when vWF is exposed to high shear stress. This is consistent with the finding that shear stress enhances the proteolysis of $\mathrm{VWF}$ in normal plasma ${ }^{21}$ and has generated the proposed model of vWF proteolysis shown in fig 4 .

\section{vWF protease activity in TTP}

Using the techniques discussed above, plasma derived from patients with both congenital and sporadic TTP has been tested in vitro for vWF cleaving protease activity because defective vWF multimeric processing could explain many of the above findings. Initially, a group of four patients with chronic relapsing TTP were shown to lack vWF metalloproteinase activity in the absence of inhibitor. ${ }^{22}$ More recently, a total of 53 patients, 30 with TTP and 23 with HUS, were studied. Again, a lack of vWF cleaving activity was found in six individuals with familial TTP without evidence for a concomitant inhibitor. Of 24 with non-familial TTP, 20 had severe and four moderate protease deficiency. In only five of 15 such patients studied was there complete recovery of $\mathrm{vWF}$ cleaving activity with normalisation of the platelet count. Furthermore, in five of the remaining patients, protease activity was evident only after weeks or even months in clinical remission. An inhibitor was found in 20 subjects from this group and was of the IgG subclass in five of five cases tested. In contrast, of the 23 patients with HUS (familial and sporadic), 21 had normal values of $\mathrm{vWF}$ cleaving protease activity during the acute episode, whereas in two patients values were slightly reduced. ${ }^{23} \mathrm{~A}$ similar phenomenon has been shown independently by Tsai: 39 samples from 37 patients with acute TTP all had severe deficiency of $\mathrm{vWF}$ cleaving protease. Inhibitory activity was detected in 26 of these 39 samples, again of the IgG subclass. Sixteen patients were studied during remission: vWF protease activity had normalised and there was no evidence of persisting inhibitory antibodies. ${ }^{24}$ Thus, there is strong supporting clinical evidence that $\mathrm{vWF}$ cleaving protease activity is integral to the pathogenesis of TTP.

As a result of these findings, it has been proposed that acute single episode TTP might be an autoimmune disorder with a secondarily acquired functional deficiency of $\mathrm{vWF}$ cleaving protease. Why the metalloproteinase is selectively targeted and the cause of the transient or intermittent defect in immune regulation are unknown. If the protease were to be endothelial surface bound, protease specific antibody formation might result in endothelial cell apoptosis and hence endothelial disregulation. Certainly endothelial injury has long been considered by many to be the prime event in TTP, although the exact nature of the endothelial insult remains unclear. Thrombomodulin, ${ }^{25}$ P-selectin (GMP-140), ${ }^{26}{ }^{27}$ tissue plasminogen activator (tPA), ${ }^{28}$ and plasminogen activator inhibitor 1 (PAI-1) ${ }^{29}$ are all raised, whereas tissue factor pathway inhibitor (TFPI) ${ }^{30}$ is reduced, consistent with endothelial damage. These observations of endothelial activation, together with histological findings of intimal proliferation, luminal stenosis, and absent 
inflammatory changes, are consistent with apoptosis. There is some in vitro evidence for apoptosis of restricted lineage microvascular but not macrovascular endothelial cell lines induced by TTP plasma. ${ }^{31}{ }^{32}$ This parallels the predilection for microvascular thrombosis within the renal and cerebral microvasculature seen clinically. Further work will be required to ascertain whether this mechanism is physiologically important.

Alternatively, should vWF cleaving protease be freely circulating, then autoantibody formation to the protease might be expected simply to result in circulating ULvWF multimers. However, as detailed above, preferential binding of such multimers to platelets under the conditions of high shear pressures that exist within the renal and cerebral microvasculature could explain the clinical features of the disease. Using flow cytometric techniques it has been shown that platelet bound vWF is raised in patients with TTP compared with normal adults. ${ }^{33}$ Concordance between vWF and P-selectin expression was not a consistent finding, suggesting that the platelet bound vWF was derived from plasma rather than the platelet $\alpha$ granules. This might explain the finding that although patients with single episode TTP classically have circulating ULvWF multimers, these can disappear as the episode continues or worsens. ${ }^{34}$ Persistence of $\mathrm{ULvWF}$ multimers into clinical remission is predictive of subsequent relapse and might reflect the continuing presence of autoantibodies and consequent impaired ULvWF processing.

In contrast, chronic relapsing TTP appears to be caused by an absolute deficiency of protease activity that might be the result of a constitutional defect in its production, survival, or activity. Again, ULvWF multimers are often detected during remission, although relapses are associated with their disappearance. Because episodes occur at three to four week intervals, it has been suggested that the progressive accumulation of ULvWF multimers might periodically exceed a threshold value and precipitate platelet aggregation.

These hypotheses may, however, prove to be an oversimplification. A sibling of an index patient with congenital TTP has been identified with absent protease activity but, to date, no TTP or HUS like syndrome. ${ }^{23}$ In addition, protease activity has not been shown to recover fully in all remission cases of adult onset TTP: whether this is the result of an interacting hitherto unrecognised cofactor or because subnormal values may be efficacious is yet to be elucidated. Indeed, the protease does appear to have a uniquely long half life: calculated as between two and four days in two patients with constitutional protease deficiency. ${ }^{35}$ Moreover, metalloprotease activity was not significantly different from normal controls in eight patients studied with bone marrow transplant associated TTP. ${ }^{36}$ This might suggest that there are alternative mechanisms mediating TTP or, in fact, that bone marrow transplant associated TTP is a distinct pathological entity. Certainly, it has long been recognised that contrary to classic TTP, bone marrow transplant associated TTP rarely responds to plasma exchange procedures.

Despite these exceptions, the evidence at present suggests that $\mathrm{VWF}$ cleaving protease activity plays a central role in the pathogenesis of the major subtypes of TTP. Precise identification and purification of this $\mathrm{vWF}$ cleaving protease activity is now required to investigate its pathological role in greater depth. It is hoped that this will result in further advances in treatment and improve the prognosis of this potentially fatal disease.

1 Moschowitz E. Hyaline thrombosis of the terminal arterioles and capillaries: a hitherto undescribed disease. Proceedings of the New York Pathological Society 1924;24:21-4.

2 Shumak KH, Rock GA, Nair RC, Members of the Canadian Apheresis Group. Late relapses successfully treated for thrombotic thrombocytopenic purpura. Ann Intern Med 1995;122:569-72.

3 Bennett CL, Weinberg PD, Rozenberg-Ben-Dror K, et al. Bennett CL, Weinberg PD, Rozenberg-Ben-Dror K, et al.
Thrombotic thrombocytopenic purpura associated with Thrombotic thrombocytopenic purpura as
ticlopidine. Ann Intern Med 1998;128:541-4.

4 Tarantolo ST, Landmark JD, Iwen PC, et al. Bartonella-like Tarantolo ST, Landmark JD, Iwen PC, et al. Bartonella-like
erythrocyte inclusions in thrombotic thrombocytopenic purpura. Lancet 1997;350:1602

5 Chintagumpala MM, Hurwitz RL, Moake JL, et al. Chronic relapsing thrombotic thrombocytopenic purpura in infants with large von Willebrand factor multimers during remission. F Pediatr 1992;120:49-53.

6 Harkness DR, Byrnes JJ, Lian EC-Y, et al. Hazard of platelet transfusion in thrombotic thrombocytopenic purpura. ҰAMA 1981;246:1931-3.

7 Asada Y, Sumiyoshi A, Hayashi T, et al. Immunohistochemistry of vascular lesion in thrombotic thrombocytopenic purpura, with special reference to factor VIII related purpura, with special reference to
antigen. Thromb Res 1985;38:469-79.

8 Moake JL, Turner NA, Stathopoulos NA, et al. Involvement of large plasma von Willebrand factor (vWF) multimers and unusually large vWF forms derived from endothelial cells in shear stress-induced platelet aggregation. F Clin Invest 1986;78:1456-61.

9 Moake JL, Turner NA, Stathopoulos NA, et al. Shearinduced platelet aggregation can be mediated by vWF released from platelets, as well as by exogenous large or unusually large vWF multimers, requires adenosine diphosphate, and is resistant to aspirin. Blood 1988;71: 1366-74.

10 Peterson DM, Stathopoulos NA, Giorgio TD, et al. Shear-induced platelet aggregation requires von Will-
ebrand factor and platelet membrane glycoproteins Ib and IIb-IIIa. Blood 1987;69:625-8.

11 Moake J, Chintagumpala M, Turner N, et al. Solvent/ detergent-treated plasma suppresses shear-induced platelet aggregation and prevents episodes of thrombotic thrombocytopenic purpura. Blood 1994;84:490-7.

12 Karpman D, Lethagen S, Kristoffersson A-C, et al. Von Willebrand factor mediates increased platelet retention in recurrent thrombotic thrombocytopenic purpura. Thromb recurrent thrombotic thromb
Haemost 1997;78:1456-62.

13 Tsai H-M, Angel RL, Hatter VB, et al. The high molecular weight form of endothelial cell von Willebrand factor is released by the regulated pathway. Br F Haematol 1991;79: 239-45.

14 O'Brien JR, Etherington MD. The endothelial von Willebrand factor in venous occlusion blood. Thromb Haemost 1997;78:1528-9.

15 Zimmerman TS, Dent JA, Ruggeri ZM, et al. Subunit composition of plasma von Willebrand factor. $\mathcal{F}$ Clin Invest 1986;77:947-51.

16 Tsai H-M, Angel RL, Susan II. Subunit composition of plasma von Willebrand factor multimers: evidence for a non-proteolytic mechanism resulting in apparent increase in proteolytic fragments. Thromb Res 1991;63:179-88.

17 Dent JA, Galbusera M, Ruggeri ZM. Heterogeneity of plasma von Willebrand factor multimers resulting form plasma von Willebrand factor multimers resulting form
proteolysis of the constituent subunit. $\mathcal{F}$ Clin Invest proteolysis of the

18 Phillips MD, Moake JL, Nolasco L, et al. Plasma von Willebrand factor processing activity functions like a disulfide bond reductase: reversible decrease of multimer size [abstract]. Thromb Haemost 1993;69:2342.

19 Furlan M, Robles R, Lämmle B. Partial purification of a protease from human plasma cleaving von Willebrand factor to fragments produced by in vivo proteolysis. Blood 1996;87:4223-34.

20 Tsai H-M. Physiologic cleavage of von Willebrand factor by a plasma protease is dependent on its conformation and requires calcium ion. Blood 1996;87:4235-44.

21 Tsai H-M, Susan II, Angel RL. Shear stress enhances proteolysis of von Willebrand factor in normal plasma. Blood 1994;83:2171-9.

22 Furlan M, Robles R, Solenthaler M, et al. Deficient activity of von Willebrand factor-cleaving protease in chronic relapsing thrombotic thrombocytopenic purpura. Blood 1997;89:3097-103. 
23 Furlan M, Robles R, Galbusera M, et al. Von Willebrand factor-cleaving protease in thrombotic thrombocytopenic purpura and the hemolytic-uremic syndrome. $N$ Engl $f$ Med 1998;339:1578-84.

24 Tsai H-M, Lian EC-Y. Antibodies to von Willebrand factorcleaving protease in acute thrombotic thrombocytopenic purpura. N Engl f Med 1998;339:1585-94.

25 Wada H, Mori Y, Shimura M, et al. Poor outcome in disseminated intravascular coagulation or thrombotic thrombocytopenic purpura patients with severe vascular endothelial cell injuries. Am f Hematol 1998;58:189-94.

26 Chong BH, Murray B, Berndt MC, et al. Plasma P-selectin is increased in thrombotic consumptive platelet disorders. Blood 1994;83:1535-41.

27 Katayama M, Handa M, Araki Y, et al. Soluble P-selectin is present in normal circulation and its plasma level is elevated in patients with thrombotic thrombocytopenic purpura and haemolytic uraemic syndrome. Br F Haematol 1993;84:702-10

28 Wada H, Kaneko T, Ohiwa M, et al. Increased levels of vascular endothelial cell markers in thrombotic thrombocytopenic purpura. Am f Hematol 1993;44:101-5.

29 Anthony MT, Zeigler ZR, Lister J, et al. Plasminogen activator inhibitor (PAI-1) antigen levels in primary TTP and secondary TTP post-bone marrow transplantation. Am $\mathscr{F}$ Hematol 1998;59:9-14.
30 Kobayashi M, Wada H, Wakita Y, et al. Decreased plasma tissue factor pathway inhibitor levels in patients with tissue factor pathway inhibitor levels in patients with
thrombotic thrombocytopenic purpura. Thromb Haemost thrombotic thron

31 Laurence J, Mitra D, Steiner M, et al. Plasma from patients with idiopathic and human immunodeficiency virusassociated thrombotic thrombocytopenic purpura induces apoptosis in microvascular endothelial cell lines. Blood 1996;87:3245-54.

32 Mitra D, Jaffe EA, Weksler B, et al. Thrombotic thrombocytopenic purpura and sporadic hemolytic-uremic syndrome plasmas induce apoptosis in restricted lineages of human microvascular endothelial cells. Blood 1997;89:1224-34.

33 Chow TW, Turner NA, Chintagumpala M, et al. Increased von Willebrand factor binding to platelets in single episode and recurrent types of thrombotic thrombocytopenic purpura. Am ₹ Hematol 1998;57:293-302.

34 Moake JL, McPherson PD. Abnormalities of von Willebrand factor multimers in thrombotic thrombocytopenic purpura and the hemolytic-uremic syndrome. Am $\mathcal{f}$ Med purpura and the

35 Furlan M, Robles R, Morselli B, et al. Recovery and half-life of von Willebrand factor-cleaving protease after plasma therapy in patients with thrombotic thrombocytopenic purpura. Thromb Haemost 1999;81:8-13.

36 Van der Plas RM, Schiphorst ME, Huizinga EG, et al. von Willebrand factor proteolysis is deficient in classic, but not in bone marrow transplantation-associated, thrombotic thrombocytopenic purpura. Blood 1999;93:3798-802

\section{PATHOLOGY INTERACTIVE 2000}

Do you know ... ?

Pathology Interactive Volume 2 now offers:

Up to three CPD credits per review article

1 credit for reading the article and completing the associated questions

1 credit for additional reading, following up references

1 credit for making notes, identifying learning outcome or identifying further learning needs

CPD credits for working on articles outside your specialty

\section{Pathology Interactive Volume 2 Issue 1 includes articles in:}

Microbiology

Histopathology

Molecular pathology

Further issues in $\mathbf{2 0 0 0}$ will cover further articles in these specialties, plus:

Chemical pathology

Haematology

Immunology

Including case study and picture quiz formats

Pathology Interactive 2000 Volume 2, 4 issues (March, June, September, December) ISSN 14665743 Accredited by the Royal College of Pathologists

Subscription rate: $£ 75+$ VAT personal ${ }^{\star}, £ 150+$ VAT institutional (multiuser rate on application to the publisher).

Send orders to: BMJ Publishing Group, Journals Marketing Dept. PO Box 299, London WC1H 9TD, UK; fax credit card orders to: +44 (0)20 7383 6402; call subscriptions hotline +44(0)207383 6270; email orders to: subscriptions@bmjgroup.com

*ACP members receive Pathology Interactive with their copy of fournal of Clinical Pathology, as a membership benefit. 